$\begin{array}{lllllllllllllllllllllllllll}R & E & S & E & A & R & C & H & R & E & P & O & R & T\end{array}$

\title{
Ethnic Self-Awareness as a Function of Ethnic Group Status, Group Composition, and Ethnic Identity Orientation
}

\author{
GREG M. KIM-JU \\ RAMSAY LIEM \\ Boston College
}

\begin{abstract}
This study found that ethnic self-awareness (i.e., the extent to which people are consciously aware of their ethnicity at any given moment) has different meanings for European Americans and Asian Americans and for Asian Americans with different ethnic identity orientations. The authors found main effects of ethnic group status and ethnic composition on ethnic self-awareness when comparing Asian Americans and European Americans. There was also an interaction effect between ethnic composition and ethnic identity orientation for Asian Americans when examining ethnic self-awareness. Findings are discussed in relation to theories that predict salience of ethnicity and to educators and practitioners who deal with ethnic minority group members.

- ethnic self-awareness $\bullet$ ethnic identity $\bullet$ ethnic composition • Asian Americans
\end{abstract}

Until recently, psychological approaches to identification with ethnicity implicitly adopted the view that identity achievement, once it occurs, is largely stable (Berry, 1990; Phinney, 1990). Recent studies have suggested, however, that ethnicity may be a dy- namic, fluid part of the social self that varies as a function of the social composition of the settings in which people participate (Turner, Oakes, Haslam, \& McGarty, 1994). One aspect of this variability involves ethnic self-awareness, or the extent to which people

- Greg M. Kim-ju and Ramsay Liem, Department of Psychology, Boston College.

A portion of this article was presented at the 27th Annual Convention of the Asian American Psychological Association, Boston, August 1999.

We wish to thank the participants of this study and our research assistants Wayne Harmon, Karen Diep, and Diane Tanaka. We also wish to thank Milton Butts Jr. and Linda Tropp for their comments on earlier versions of this article.

Correspondence concerning this article should be addressed to Greg M. Kim-ju, who is now at the Department of Psychology, California State University, $6000 \mathrm{~J}$ Street, Sacramento, California 95819-6007. E-mail: kimju@csus.edu

Cultural Diversity and Ethnic Minority Psychology Vol. 9, No. 3, 289-302
Copyright 2003 by the Educational Publishing Foundation 1099-9809/03/\$12.00 DOI: 10.1037/1099-9809.9.3.289 
are consciously aware of their ethnicity at any given moment.

The present study explored stable and situational factors that influence ethnic selfawareness in interpersonal situations. Specifically, we examined (a) how majority and minority status associated with ethnic groups and the ethnic composition of interpersonal situations influence ethnic selfawareness of Asian Americans and European Americans, and (b) for Asian Americans, the extent to which ethnic selfawareness is related to ethnic identity orientation as well as the ethnic composition of interpersonal situations.

\section{Ethnic Identity Models}

Psychologists examining ethnic identity have generally treated it as an integral component of people's social identity (Bernal, Saenz, \& Knight, 1991; Berry, 1990; Phinney, 1990). They have emphasized different aspects of social identity that correspond to their theoretical orientations and specific empirical concerns. Two broad distinctions involve acculturation versus developmental approaches to ethnic identity.

\section{Acculturation Framework}

Acculturation approaches to ethnic identity focus on changes in people's attitudes, values, and behaviors resulting from contact between two cultures. The work of Berry (1990) is, perhaps, the most widely recognized and respected exemplar of this tradition. It focuses on identification with culture of origin and valuing of attachments to the new culture and, depending on a person's preferences, the four familiar resolutions to acculturation: (a) assimilation-exclusive identification with the new culture; (b) separation-exclusive identification with culture of origin; (c) integration or biculturalityidentification with both cultures; and (d) marginality - the absence of identification with either culture. The main concerns of acculturation theorists are how a person ex- periences his or her culture of origin in relation to the new culture, the factors that influence this adaptation, and the implication of acculturation for interpersonal and intergroup contact and psychological adjustment (Abe-Kim, Okazaki, \& Goto, 2001; Cuellar \& Roberts, 1997).

\section{Developmental Framework}

The developmental perspective emphasizes stage models of ethnic identity development. For example, Phinney (1990) proposed that ethnic identity develops in a three-stage progression, from (a) unexamined ethnic identity characterized by a lack of attention to ethnicity and unreflected preference for the dominant culture, to (b) ethnic identity search involving immersion in culture of origin that may involve a rejection of dominant cultural values, to (c) ethnic identity achievement characterized by an appreciation for an individual's ethnicity and resolution of conflicts with the dominant group. These models focus chiefly on the extent of a person's exploration of ethnicity and assume that overidentification with the dominant culture can have negative psychological consequences.

Both the acculturation and developmental approaches to ethnic identity implicitly assume that a person eventually achieves a largely stable resolution. Still, there is some recognition that a person's stage of development or resolution of cultural adaptation may change over time and across contexts. For example, Berry (1990) suggested that how people relate to their own and host culture may vary in different situations (e.g., school vs. home).

The belief that aspects of ethnic identity may be responsive to situational factors is consistent with a variety of general theoretical models that emphasize the interaction between person and environment to explain behavior (Rogoff, 1990). By and large, these context-sensitive approaches have demonstrated superior ability to predict behavior as compared with those relying principally on fixed person variables like traits or attitudes. 
Closer to our focus, Turner et al. (1994) have demonstrated that the salience of different aspects of social identity (e.g., social class, occupation) is responsive to the intergroup context involving characteristics of people in a given social setting, including particular features of the social self such as age, gender, or class.

From a practical point of view, assuming that ethnic identity is a constant influence on a person across situations can lead to overgeneralizations detrimental to that person. For example, a teacher who perceives an ethnic minority student as confident and at ease among peers in situations may err in assuming that the classroom is equally comfortable for that student. As we argue in this study, the ethnic composition of the group in these two contexts may be a critical factor that influences this person's experience in each setting. Similarly, clinicians seeking to be culturally sensitive by understanding a client's basic orientation to ethnic identity may overlook how a person experiences self and others differently depending on the ethnic makeup of the situation. These are not original ideas by any means, but they serve to highlight the importance of devoting more attention to situational dimensions of ethnic identity in theory and research.

\section{From Ethnic Identity to Self-Awareness}

One aspect of ethnic identity that may be especially responsive to context is its salience for the individual. For example, even though a person's identification with ethnicity may be relatively stable (e.g., during a particular stage of development), how much this aspect of social identity comes into play or is a conscious feature of self-awareness can vary according to immediate circumstances. Research that has explored this possibility has drawn on distinctiveness theory and self-awareness theory. Distinctiveness theory assumes that people are most aware of social characteristics (e.g., age, gender, ethnicity) that distinguish their self from others in a social situation (McGuire, McGuire, Child, \& Fujioka, 1978). Similarly, selfawareness theory posits that the aspect of the self to which people attend is partly determined by external events or conditions that heighten the salience of different facets of the social self (Duval \& Wickland, 1972). For example, in a classroom situation comprised of European American female students and one Asian American female student, an observer would be expected to notice the ethnicity of the Asian student. On the other hand, in a classroom in which everyone else is an Asian American male, the female gender of the same student should stand out.

Both distinctiveness theory and selfawareness theory have been used to anticipate how individuals become spontaneously aware of social characteristics related to the self. Studies of children (McGuire et al., 1978; McGuire \& Padawer-Singer, 1976) and young adults (Cota \& Dion, 1986; Mehra, Kilduff, \& Brass, 1998) in both natural (e.g., classrooms, home) and laboratory settings have demonstrated that respondents are more likely to be aware of their age, birthplace, gender, and the like when these attributes are underrepresented in these situations.

Although this research supports the view that the immediate situation can serve to cue particular features of the social self, it has two major limitations as a general approach to ethnic self-awareness. First, in conducting these studies, researchers have implicitly adopted the stance of the majority ethnic group, European Americans. Specifically, the assumption that salience of ethnicity increases when the ethnic composition of the group setting is less representative of a person's ethnicity may be valid if that person is accustomed to being in the ethnic majority, that is, the case for European Americans. For them, ethnicity may generally be "taken for granted" and only becomes an object of self-awareness during those exceptional moments when they happen to be among a large number of people from a different ethnic group, for example, being a guest at a Chinese Buddhist temple. Being in the mi- 
nority presumably confers distinctiveness and, hence, salience. This mechanism for enhancing distinctiveness may be particularly relevant to individuals whose ethnicity is customarily in the majority in everyday social settings. We believe this kind of experience in the larger society is an implicit assumption of research on ethnic selfawareness, heretofore unarticulated because the majority of participants in research of this kind have been European Americans.

For ethnic minorities, however, salience of ethnicity may be more consistent across situations, or even more pronounced when their group is in the majority. Asian Americans, for example, are typically in the minority in everyday social interactions. For them, being in the majority is itself a novel situation that may actually heighten awareness of ethnicity. Recent studies have reported results not predicted by these theories in which ethnic minority groups such as African Americans and Hispanic Americans were more aware of their ethnicity when in the ethnic majority than in the ethnic minority (Aries et al., 1998; Dutton, Singer, \& Devlin, 1998; Hardiman, \& Jackson, 1992). These findings suggest that when the overall status of an ethnic group in the wider society is taken into consideration, the general applicability of distinctiveness theory and selfawareness theory is called into question.

\section{Ethnic Identity Orientation as a Factor in Ethnic Self-Awareness}

Focusing on the experience of the dominant ethnic group in the wider society and interpreting findings in terms of extant theory based on the majority perspective, researchers have paid little attention to how a person's identification with ethnicity might influence ethnic self-awareness in different situations. Reconnecting the issue of situational aspects of ethnicity to the dominant line of research on how people are oriented to their ethnicity raises the possibility that different orientations and other variables such as the ethnic composition of a situation may have a bearing on ethnic self-awareness. For example, we would generally expect Chinese Americans to become aware of their ethnicity in situations in which they are in the ethnic minority. This pattern of self-awareness, however, may be particularly valid for Chinese Americans in the ethnic identity search stage because they are actively exploring their ethnicity and attitudes toward their own group and the dominant White group. They may be self-consciously identified more with the Chinese or Chinese American culture than the dominant culture and are, in theory, less comfortable in situations with dominant group members. These individuals may, therefore, be especially aware of their ethnicity in the presence of a majority of people from the dominant group. However, Chinese Americans in the unexamined stage might react differently in the same situation because of their positive attitudes and beliefs about the dominant group. These individuals identify strongly with the dominant culture and feel comfortable in the presence of dominant group members and, therefore, may not be as self-conscious of their ethnicity in this situation. Hence, a second important factor influencing the salience of ethnicity, in addition to the majority-minority group status, may be ethnic identity orientation as reflected in a person's stage of ethnic identity development.

\section{The Present Study}

This study examined two issues. First, we focused on the influence of ethnic group status in the wider society (i.e., dominant/ European American vs. minority/Asian American ethnic group) and the ethnic composition of social situations on ethnic self-awareness. Second, we looked at how awareness of ethnicity might vary for Asian Americans with different ethnic identity orientations in social situations with varying ethnic compositions.

In our first set of analyses, we expected that European Americans would display 
greater awareness of their ethnicity in situations in which they were in the minority in relation to other ethnic groups. In contrast, we expected Asian Americans to display heightened levels of awareness of their ethnicity in both minority and majority settings. How each group would respond to a mixed ethnic situation was treated as an empirical question.

We next expected that, among Asian Americans, ethnic identity orientation and the ethnic composition of a social gathering would both influence ethnic self-awareness. The exact pattern of responses could not be anticipated but, in general, individuals with an unexamined ethnic identity orientation were expected to exhibit comparatively low awareness of ethnicity across situations, salience being strongest when they were in the ethnic majority. Individuals with a search ethnic identity orientation were anticipated to be most conscious of their ethnicity across situations in relation to the other respondents. In view of the individualized resolutions of ethnic identity associated with an achieved orientation envisioned by Phinney (1990), it was difficult to anticipate how this group would respond to the different social scenarios.

\section{Method}

\section{Participants}

Eighty-two people (56 female and 26 male) ranging in age from 17 to 30 years old $(M=$ 20.69 years, $S D=2.65$ ) volunteered to participate in this study. Each participant was from the Boston area. Fifty-eight were Asian Americans (39 female and 19 male) and 24 were European Americans ( 17 female and 7 male). The Asian American participants included Chinese $(n=18)$, Filipino $(n=12)$, Indian $(n=6)$, Japanese $(n=3)$, Korean $(n$ $=16)$, Laotian $(n=1)$, and Vietnamese $(n=$ 2). Twenty-seven of these participants were born in another country but emigrated to the United States at an early age (1.5 generation), and 31 were born in the United States (second generation or higher). All of the European American participants were second generation or higher. Education and socioeconomic levels did not differ between the two principal groups. Two Asian American participants from the initial research pool were later excluded from all analyses because their responses to several measures were incomplete.

\section{Procedure}

The research protocol was administered to each participant, individually, at that participant's college or university in a room previously selected for this purpose. Participants were told that for the next 30 min they would complete several questionnaires in folders consisting of different scenarios. They were instructed to read a description of the purpose of the study, invited to ask questions, and then asked to sign a consent form. The researcher then gave the participants three folders containing the vignette questionnaire (see Measures section) and provided them with the following instructions:

As you look at each picture and read its description, imagine yourself in the social interaction that is pictured and described. Then answer the questions that follow the picture and vignette. After you have responded to the picture and description in the first folder, go on to the second, and then the third. However, complete the questions for each scene before moving onto the next.

The researcher addressed any questions and informed the participant that he would return in $30 \mathrm{~min}$. He then left the room. After the participant completed the vignette questionnaire, the researcher administered two questionnaires that included an ethnic identity measure and several demographic items.

The researcher debriefed the participants and answered any questions. In exchange for their participation, participants received 2 research credits toward a course requirement, were entered into a drawing for $\$ 50$ gift certificates to a bookstore, or were given a $\$ 10$ incentive. 
Half of the participants were received by a White male researcher and the other half by an Asian American male researcher to control for possible influences of the researcher's ethnicity on participants' reactions to the measures. No experimenter effects were found.

\section{Measures}

Vignette Questionnaire. This instrument used three social situations commonly encountered by college students. They were generated from an open-ended interview with an independent sample of 15 European American and Asian American students asked to describe common situations in which they were likely to encounter few, some, or many peers from their own ethnic group. The three settings were a school cafeteria, a classroom, and a dormitory lounge.

Each vignette included a picture of a group of six students taken in one of the settings and a brief description of the action (see Table 1). The groups comprised equal numbers of male and female students. To vary the ethnic group composition of the vignettes, we designed a minority, mixed, and majority ethnic composition for each picture/narrative by photographing European American and Asian American volunteers staged according to the described action. Minority and majority compositions in- volved all people of the same ethnic group. Thus, for an Asian American respondent, the minority composition was comprised of all European Americans and the majority, all Asian Americans. For European American participants, the pattern was reversed. Minority composition pictures for Asian Americans, thus, served as majority composition scenes for European Americans, and vice versa. The mixed condition involved equal numbers of respondents' own and other ethnic group members. Three scenarios were used to avoid context dependence in the assessment of ethnic selfawareness. Altogether, nine scenarios were designed for this research. Each participant responded to the three settings, one of which contained the minority ethnic composition, another the mixed, and the third, the majority. The order of these settings was randomized. The ethnic composition associated with a particular scenario was also randomized across participants.

Ethnic Self-Awareness. Following exposure to each vignette, participants were asked to answer the question "Who am I?" (WAI) five times as they imagined themselves in the scene. The WAI inventory (Kuhn \& McPartland, 1954) served as the primary measure of awareness of ethnicity following the procedure of Rhee, Uleman, Lee, and Roman (1995). In this approach,

TABLE 1 Narratives of Vignette Compositions

\begin{tabular}{|c|c|}
\hline Social setting & Vignette \\
\hline Cafeteria & $\begin{array}{l}\text { You are going to have lunch at the cafeteria on your campus. You enter the cafeteria and select } \\
\text { your food. After you pay for your food, you look around for a place to sit. You see an open } \\
\text { seat and walk over to a table with a group of students you do not know. You sit down and } \\
\text { begin speaking to several people near you as you eat your lunch. }\end{array}$ \\
\hline Classroom & $\begin{array}{l}\text { You are starting a new semester. You are going to class. As you enter your classroom, you look } \\
\text { around and see that all of the students are strangers. You sit down and listen to the teacher. } \\
\text { After several minutes, your teacher asks everyone to get into small groups in order to break } \\
\text { the ice and discuss the first assignment. You join the group that is forming near you. You } \\
\text { look around and then join the discussion with your group members. }\end{array}$ \\
\hline Dormitory & $\begin{array}{l}\text { You have received an invitation to a dorm party from one of your new classmates. You enter the } \\
\text { dorm and walk to the lounge where the party is being held. Your new classmate sees you and } \\
\text { welcomes you in. As you enter the lounge, you don't see anyone you know, but you approach } \\
\text { several people and begin to talk. }\end{array}$ \\
\hline
\end{tabular}


ethnic salience is operationalized as the percentage of WAI responses that refer to ethnicity and requires coding of all responses as ethnicity related or unrelated. Two independent judges used the following guidelines to conduct this coding:

1. Ethnicity related. This included references to the following: pan-ethnicity (e.g., Asian, Caucasian), ethnicity (e.g., Chinese American, Korean American), nationality (e.g., American, Filipino), origin outside of the United States (e.g., "I am from Hong Kong" or "I was born in the United States"), and others (e.g., ethnic minority, minority, majority, and different).

2. Ethnicity unrelated. This included all other responses (e.g., "I am tall" or "I am Keith"). Self-descriptions that referred to unspecified differences (e.g., "I am different") were also coded as ethnicity related only if respondents also referred to their ethnicity explicitly in another response. All other self-descriptions were classified as ethnicity unrelated.

A total ethnic self-awareness score was determined using the procedure of Rhee et al. (1995) that codes all discrete references to ethnicity in each of the five responses to the WAI as a percentage. Respondents' percentage ethnicity-related scores were used in all subsequent analyses to operationalize self-awareness of ethnicity. For example, if participants mentioned ethnicity once in response to each of the three vignettes, then they were given an ethnicity-related score of $20 \%$ for each vignette (participants gave five responses to each vignette). If participants mentioned ethnicity three times in one vignette but not at all to the other two vignettes, they received ethnicity-related scores of $60 \%$ for one vignette and $0 \%$ for each of the other two vignettes. Greg M. Kim-ju and one undergraduate research assistant who was unaware of the specific hypotheses of this study coded all WAI responses independently and achieved a
92\% agreement on coding assignments of responses as ethnicity related or ethnicity unrelated.

Preliminary analyses of scores on ethnic self-awareness in relation to the key demographic variables of socioeconomic status, gender, generation in the United States, age, and Asian ethnic group (those with a sample size of at least 12, Chinese, Filipino, and Koreans) were conducted. None of these variables were significantly related to ethnic self-awareness.

Ethnic Identity Orientation. Ethnic identity orientation was measured using the Multigroup Ethnic Identity Measure (MEIM) developed by Phinney (1992). This measure was specifically designed for use with different ethnic groups. The MEIM consists of 14 items assessing three general domains: ethnic identity achievement, which includes issues related to exploration and resolution (7 items; e.g., participants have a clear sense of their ethnic background and what it means for them); positive ethnic attitudes and sense of belonging (5 items; e.g., participants are happy that they are members of the group to which they belong); and ethnic practices or behaviors (2 items; e.g., participants take part in cultural practices of their own group, such as special food, music, or customs). Items are rated using a 4-point rating scale ranging from strongly agree to strongly disagree. Scores are calculated by reversing negatively worded items, summing across items, and calculating the overall mean score. The MEIM was not designed specifically as a measure of ethnic identity stages. However, in the absence of any published alternative, we based our operationalization of ethnic identity orientation on the overall distribution of MEIM scores in our Asian American sample that produced a trimodal distribution and on personal communication with Jean Phinney (March 5, 1999). We consequently treated scores in the bottom tertial as indicating the unexamined orientation ( $n=18, M=2.59)$, high scores as indicating the achieved orientation $(n=20$, $M=3.80$ ), and mid-range scores as indicat- 
ing the search identity orientation $(n=18$, $M=3.25)$.

Some support for the validity of this procedure is provided by the fact that age and generation were significantly related to ethnic identity orientation $(r=.27, p<.05$ and $r=-.30, p<.05$, respectively; Moyerman \& Forman, 1992). As expected, individuals in the unexamined ethnic identity orientation group were younger than those whose orientation was search or achieved. In addition, more multigenerational Asian Americans were represented in the unexamined ethnic identity orientation group than in the search and achieved ethnic identity orientation groups. In contrast, tests of association between ethnic identity orientation and gender, socioeconomic status, and ethnic group were also performed but, as expected, were not significant. Although not definitive, these findings suggest that our method for grouping Asian American participants according to the three ethnic identity orientations was reasonable.

Reliability coefficients (Cronbach's alpha) for the total score and for exploration, sense of belonging, and ethnic behavior subscales were $.90, .82, .86$, and .49 , respectively, and are consistent with the range of reliability estimates published on specific Asian American samples ranging from adolescence to early adulthood (Lee, Falbo, Doh, \& Park, 2001). The MEIM is generally regarded to have acceptable construct validity. Prior research has demonstrated that respondents with higher ethnic identity scores based on the MEIM tended to have higher self-esteem (Phinney \& Alipuria, 1996).

\section{Results}

\section{Ethnic Self-Awareness: Asian Americans and European Americans}

To test ethnic self-awareness in the context of varying compositions of ethnic group members for Asian Americans and European Americans, we compared ethnicity responses to the WAI questions following participants' exposure to each of three vignettes differing in ethnic composition. A $2 \times 3$ mixed analysis of variance (ANOVA) with ethnic group (European American vs. Asian American) as a between-subjects variable and vignette composition (minority vs. mixed vs. majority), a repeated measures variable, and ethnic self-awareness, the primary dependent variable, was used to conduct these comparisons.

As anticipated, results revealed that Asian Americans experienced ethnic selfawareness differently in response to these vignettes than did European Americans. There was a main effect for ethnic group, $F(1,78)=13.95, p<.001$, in which Asian Americans were more aware of their ethnicity across settings $(M=16.76 \%)$ than were European Americans $(M=4.65 \%)$. Moreover, we found an Ethnic Group $\times$ Vignette Composition interaction, $F(2,156)=$ $6.14, p<.001$. Rather than being less aware of their ethnicity in the majority composition compared with the other vignette ethnic compositions, ethnicity was equally salient for Asian Americans in response both to the minority $(M=17.55 \%), t(55)=2.44, p<.05$, and majority $(M=21.46 \%), t(55)=3.89, p<$ .001 , vignette compositions but less so to the mixed vignette composition $(M=11.27 \%)$.

In contrast, European Americans were more aware of their ethnicity in response to the minority vignette composition $(M=$ $10.63 \%)$ than the mixed $(M=3.33 \%), t(23)$ $=2.26, p<.05$, and majority $(M=0 \%), t(23)$ $=2.77, p<.01$, vignette compositions. European Americans were also more aware of their ethnicity in the mixed vignette composition than in the majority vignette composition, $t(23)=2.14, p<.05$.

Differences in ethnic self-awareness between Asian Americans and European Americans were greatest in the expected direction in the majority vignette composition, $t(78)=4.57, p<.001$, followed by the mixed vignette composition, $t(78)=2.33$, $p<.05$. The difference was only marginally significant for the minority composition scenarios, $t(78)=1.57, p=.12$. This pattern of relationships is illustrated in Figure 1. 


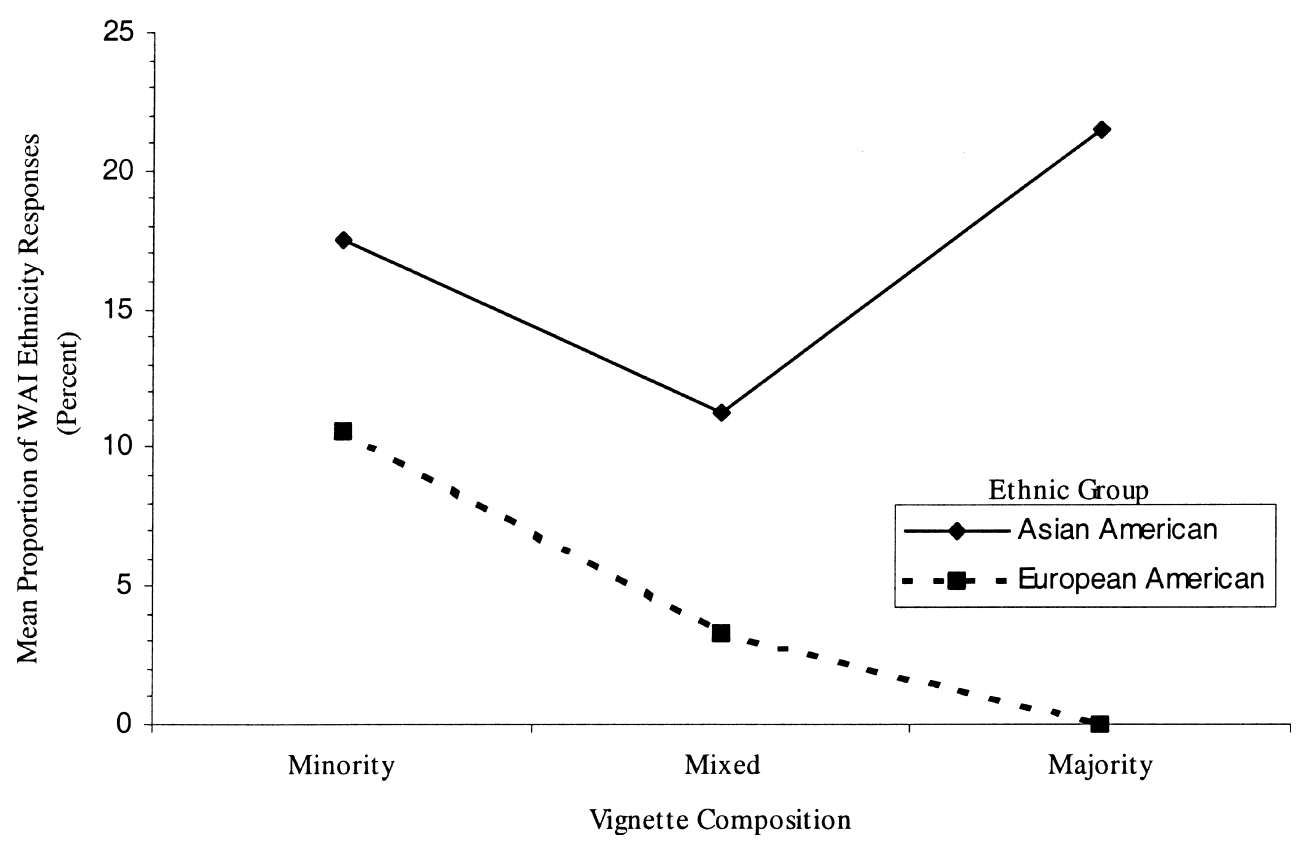

Figure 1. Mean proportion of "Who Am I?" (WAI) responses referring to ethnicity by ethnic group and vignette composition. Mean ethnic self-awareness scores across ethnic groups (European Americans vs. Asian Americans) within vignettes (minority vs. mixed vs. majority) are presented. Unweighted means are used for ethnic self-awareness scores for European Americans and Asian Americans because of the unequal sample sizes.

Unexpectedly, we also found a main effect for the vignette composition variable, $F(2,156)=4.30, p<.05$, such that awareness of ethnicity for all participants was greatest in response to the minority $(M=15.48 \%)$, $t(79)=3.23, p<.01$, and majority $(M=$ $15.02 \%), t(79)=3.05, p<.01$, vignette compositions compared with the mixed vignette composition $(M=8.88 \%)$. We found no overall differences between minority and majority vignette compositions.

Overall, these findings suggest that the ethnic composition of the social settings represented in these vignettes was related to awareness of ethnicity. More importantly, however, the pattern of these relationships was clearly dependent on the ethnicity of the respondent.

\section{Ethnic Self-Awareness Among Asian Americans}

As with our comparisons of Asian Americans and European Americans, we used WAI re- sponses to the vignette scenarios to operationalize self-awareness of ethnicity for these analyses. A $3 \times 3$ mixed ANOVA was used to explore ethnic self-awareness of Asian Americans with different ethnic identity orientations. The between-subjects variable was ethnic identity orientation (unexamined vs. search vs. achieved), and the repeated measures variable was ethnic composition of social setting (minority vs. mixed vs. majority). Ethnic self-awareness was again the primary dependent measure.

Our analyses produced a mixed picture in view of our expectations. We failed to find a significant main effect for ethnic identity orientation. However, a significant Ethnic Identity Orientation $\times$ Vignette Composition interaction, $F(4,106)=2.68, p<.05$, revealed differences in the way respondents with different ethnic identity orientations responded across the three vignette compositions, especially differences in ethnic selfawareness between individuals with search 
and achieved ethnic identity orientations. Planned comparisons indicated that ethnic self-awareness was heightened in the minority $(M=24.72 \%), t(17)=3.77, p<.01$, and majority $(M=23.17 \%), t(17)=3.58, p<.01$, vignette compositions but lower in the mixed vignette composition $(M=6.66 \%)$ for individuals with a search ethnic identity orientation. We found no differences in the way individuals with an unexamined ethnic identity orientation reacted to the three vignette compositions. Ethnic self-awareness was equally salient in the minority, mixed, and majority compositions for this group.

Although we did not have predictions regarding people with an achieved ethnic identity orientation, this group of individuals displayed greater awareness of ethnicity in response to the majority vignette composition $(M=22.35 \%)$ compared with the mixed vignette composition $(M=11.55 \%)$, $t(19)=2.38, p<.05$, as illustrated in Figure 2 . There were marginal differences between the majority and minority vignette compositions $(M=14.00 \%)$ at $p=.12$ but no differ- ences between the mixed and minority vignette compositions.

In addition to these findings, the analyses revealed an overall effect of vignette composition on ethnic self-awareness, $F(2$, $106)=7.50, p<.001$, such that all Asian American participants were more aware of their ethnicity in the majority $(M=21.46 \%)$, $t(55)=3.89, p<.001$, and minority $(M=$ $17.55 \%), t(55)=2.44, p<.05$, vignette compositions than in the mixed vignette composition $(M=11.27 \%)$. No difference was found between the majority and minority vignette compositions.

\section{Discussion}

Ethnic Self-Awareness Among Asian Americans and European Americans

As we anticipated, Asian Americans displayed a pattern of ethnic self-awareness consistent with their overall status in the society as minority group members. Collec-

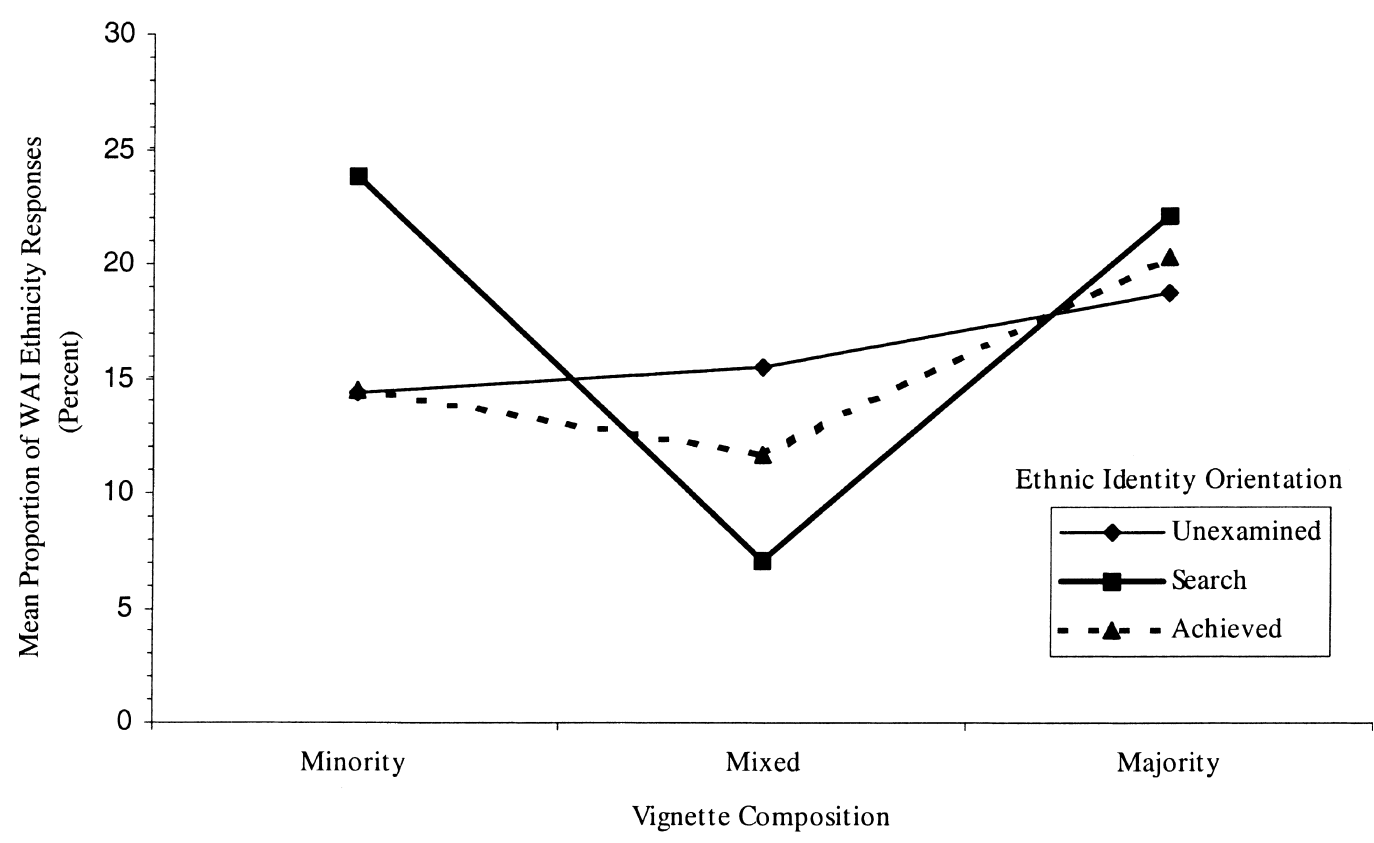

Figure 2. Mean proportion of "Who Am I?" (WAI) responses referring to ethnicity by ethnic identity orientation and vignette composition. Mean ethnic self-awareness scores across ethnic identity orientation groups (unexamined vs. search vs. achieved) within vignettes (minority vs. mixed vs. majority) are presented. 
tively, the Asian American participants not only appeared to be more aware of their ethnicity across the three ethnic compositions represented in the vignettes than were $\mathrm{Eu}$ ropean Americans but also expressed greatest ethnic self-awareness in response to the majority vignette. In contrast, European Americans responded to this scenario with virtually no awareness of their ethnicity but reported greater salience in the minority situation.

To understand these differences in ethnic self-awareness between Asian Americans and European Americans, we need to bear in mind the status of each ethnic group in the wider society. As the dominant ethnic group in the United States, European Americans may rarely encounter situations in which their taken-for-granted ethnicity is called into question (Phinney \& Alipuria, 1996). Hence, the uniqueness of being in the ethnic minority for them may be a prerequisite for distinctiveness to enhance awareness of ethnicity.

For Asian Americans, however, being in both the ethnic minority and ethnic majority, interpersonally, may represent "distinctiveness," but in different ways-in the former case, as the underrepresented group, but in the latter, as a rarely encountered social experience. Given the predominantly European American student populations on the campuses from which our Asian American participants were drawn, distinctiveness of both kinds is a likely scenario. From this perspective, it is not surprising that Asian Americans may generally be more aware of their ethnicity than are European Americans and that settings in which they are in the majority also heighten ethnic selfawareness. These as well as other recent findings (Aries et al., 1998), therefore, may warrant a modification of theories that have been used to explain salience of ethnicity such as distinctiveness theory at the very least to consider the types of daily experiences that people are likely to encounter in society in light of the demographics and sociopolitical positioning of their ethnic group. Interestingly, this study has also shown that mixed ethnic compositions elicit the least ethnic self-awareness for ethnic minority group members.

\section{Ethnic Self-Awareness and Asian American Ethnic Identity Orientation}

Our expectations for Asian Americans with different ethnic identity orientations placed in social settings with varying ethnic composition were only partially confirmed by our findings, which revealed a significant Ethnic Identity Orientation $\times$ Vignette Composition interaction, $F(4,106)=2.68, p<.05$, but not necessarily in the directions that we had predicted. Simple effects tests revealed that much of the variability in ethnic selfawareness was accounted for by individuals with a search ethnic identity orientation, followed by those with an achieved ethnic identity orientation. Of the three vignette compositions, the mixed vignette composition appeared to have elicited the least awareness of ethnicity for all participants, regardless of ethnic identity orientation.

Although individuals with a search ethnic identity orientation displayed the most variation of the three ethnic identity groups, their apparent low awareness of ethnicity in the mixed vignette composition is surprising given that we had viewed these individuals as deeply immersed in their culture of origin in their attempts to reconcile unresolved issues related to their ethnicity. One explanation for this inconsistency may be our overestimation of the degree to which such individuals are actively involved in or thinking about their ethnicity. Instead, conditions in which there is significant representation of a person's ethnic group and in which people are actively engaged may provide a modicum of safety for Asian Americans, lessening the salience or expression of ethnicity; that is, the need to be "on guard" is lessened.

The pattern displayed by the unexamined ethnic identity orientation group was most consistent with our predictions. Although there were no significant differences in ethnic self-awareness in relation to the 
three vignette compositions for this group, there was a trend for these participants to become more aware of their ethnicity with greater representation of their ethnic group. This pattern does not conform to distinctiveness theory or self-awareness theory, which would suggest less awareness of ethnicity as the number of people from a person's own ethnic group increases. However, if we understand this group to be highly assimilated to mainstream, European American culture (i.e., culturally they are virtually like European Americans), then this pattern conforms to the expectations of distinctiveness theory and self-awareness theory.

Participants with an achieved ethnic identity orientation reported lower levels of ethnic self-awareness in the minority scenario compared with the majority scenario. This finding suggests that such individuals may have resolved important issues related to their ethnicity but nonetheless still pay attention to or are aware of their ethnicity. It may be the case that even though individuals with an achieved ethnic identity orientation are generally comfortable with their ethnicity and function with little difficulty in various culturally and ethnically diverse situations, they are not accustomed to situations with a large representation of Asian Americans and, therefore, display heightened ethnic self-awareness in these settings. Conversely, individuals with this ethnic identity orientation may be comfortable enough with their ethnicity to approach situations with a high concentration of European Americans with confidence.

Overall, although our findings for ethnic self-awareness for respondents with an unexamined ethnic identity orientation were largely as anticipated, the pattern of ethnic self-awareness was not consistent with our predictions for those with either a search or an achieved ethnic identity orientation. While the lack of any clear findings may suggest that we may have misinterpreted the meanings of search and achieved ethnic identity orientations, it may also signify problems with the operationalization of these stances (see Methodological Limitations below).

The strongest finding regarding ethnic self-awareness involved vignette composition independent of respondents' ethnic identity orientation; all of the participants, regardless of ethnic identity orientation, displayed heightened awareness in response to the majority vignette composition. As noted earlier, this type of situation may not be the normative experience for many Asian Americans, and therefore may be especially striking for them. Outside of one or two locations on campus, such as a student union, which at times may have an unusually high representation of Asian Americans, most Asian American participants may typically not participate in or observe social settings with predominantly Asian Americans.

\section{Methodological Limitations}

Our operationalization of the ethnic identity orientations with the MEIM (Phinney, 1992) may have been less than ideal. Although used extensively in ethnic identity research, it is possible that this measure may not have captured participants' ethnic identity orientation accurately. In light of our findings that minority group status has a strong bearing on how Asian Americans experience their ethnicity, it is reasonable to suspect that people's increasing understanding of their group's social status may influence greater awareness of ethnicity. However, how people relate to being an ethnic minority is not explicitly captured in the MEIM, and hence may have led to some divergent findings at least with ethnic identity orientation. A more complete assessment of ethnic identity orientation may require items that capture the degree of sophistication of people's sociopolitical stance toward their ethnicity.

Even assuming that the MEIM is a valid measure of ethnic identity orientation, our operationalization of the three ethnic identity orientations may have been problematic. Overall, there was limited variability in MEIM scores in this sample (range $=1.93-$ 
$4.00, S D=0.53)$ and a preponderance of high scores $(M=3.23)$ due, perhaps, to the inherent homogeneity in college student populations. Although our groups did vary in expected ways on two demographic variables (generation in the United States and age of participant), their absolute scores on the MEIM suggest that they may not have been the best examples of Phinney's three ethnic identity orientations. Future research samples clearly need to be more diverse to ensure that individuals with a truly wide range of ethnic identity orientations are included.

Another limitation of this study was its focus on global factors such as majority versus minority ethnic group status and ethnic identity orientation in relation to ethnic selfawareness. To deepen our understanding of conditions that promote or mitigate ethnic self-awareness, additional research should examine potential moderating influences of such related variables as acculturation, generation, and gender. Furthermore, as with many studies of ethnic identity, this research sample was heavily weighted in the direction of younger and college-attending participants. How ethnic self-awareness is influenced by ethnic identity orientation and situational factors among older people more accustomed to the ecology of the workplace deserves exploration on its own terms. Ideally, such research, conducted by multiple investigators, will enable replication of findings with specific Asian American ethnic groups.

\section{Conclusion}

Despite these limitations, this research offers some meaningful contributions to our understanding of ethnic self-awareness. Most notably, our findings call into question the adequacy of theories that anticipate the conditions under which minorities become sensitized to their ethnicity. Whereas past research has used predominantly Caucasian samples and, therefore, inadvertently adopted the stance of the majority ethnic group, we have demonstrated that ethnic minorities such as Asian Americans react differently to social situations than as anticipated by distinctiveness theory and selfawareness theory. We also identified both relatively stable (ethnic identity orientation) and situational (ethnic composition of a social setting) factors that influence salience of ethnicity for Asian Americans, adding complexity to our understanding of how ethnic features of social identity play out in everyday life.

These findings should alert practitioners who work with diverse groups to contextual factors that affect the experience of ethnic minorities. They also suggest that change at the institutional level may be essential to meeting the needs of individuals. For example, to achieve balanced ethnic representation in classrooms which, from our findings, appears to be desirable for many participants, we would need to revisit college admissions policies that currently restrict the use of ethnic minority status as a legitimate selection consideration.

It is clear, however, that more precise understanding of the factors that determine ethnic salience and, in turn, the implications of such self-awareness on other psychological processes require further exploration using methodological as well as conceptual refinements suggested by this research. Our hope is that others will be encouraged to contribute to this effort.

\section{References}

Abe-Kim, J., Okazaki, S., \& Goto, S. (2001). Unidimensional versus multidimensional approaches to the assessment of acculturation for Asian American populations. Cultural Diversity and Ethnic Minority Psychology, 7, 232-246.

Aries, E., Olver, R., Blount, K., Christaldi, K., Fredman, S., \& Lee, T. (1998). Race and gender as components of the working selfconcept. Journal of Social Psychology, 138, 277-290.

Bernal, M., Saenz, D., \& Knight, G. (1991). Ethnic identity and adaptation of Mexican American youths in school settings. Hispanic Journal of Behavioral Sciences, 13, 135-154. 
Berry, J. (1990). Psychology of acculturation: Understanding individuals moving between cultures. In R. W. Brislin (Ed.), Applied crosscultural psychology (pp. 232-253). Newbury Park, CA: Sage.

Cota, A., \& Dion, K. (1986). Salience of gender and sex composition of ad hoc groups: An experimental test of distinctiveness theory. Journal of Personality and Social Psychology, 50, 770-776.

Cuellar, I., \& Roberts, R. (1997). Relations of depression, acculturation, and socioeconomic status in a Latino sample. Hispanic Journal of Behavioral Sciences, 19, 230-238.

Dutton, S., Singer, J., \& Devlin, A. (1998). Racial identity of children in integrated, predominantly White, and Black schools. Journal of Social Psychology, 138, 41-53.

Duval, S., \& Wickland, R. (1972). A theory of objective self-awareness. San Diego, CA: Academic Press.

Hardiman, R., \& Jackson, B. (1992). Racial identity development: Understanding racial dynamics in college classrooms and on campus. In M. Adams (Ed.), Promoting diversity in college classrooms (pp. 21-37). San Francisco: Jossey-Bass.

Kuhn, M., \& McPartland, T. (1954). An empirical investigation of self-attitudes. American Sociological Review, 19, 58-76.

Lee, R., Falbo, T., Doh, H., \& Park, S. (2001). The Korean diasporic experience: Measuring ethnic identity in the United States and China. Cultural Diversity and Ethnic Minority Psychology, 7, 207-216.

McGuire, W., McGuire, C., Child, P., \& Fujioka, T. (1978). Salience of ethnicity in the spontaneous self-concept as a function of one's ethnic distinctiveness in the social environment. Journal of Personality and Social Psychology, 36, 511-520.

McGuire, W., \& Padawer-Singer, A. (1976). Trait salience in the spontaneous self-concept. Journal of Personality and Social Psychology, 33, 743-754.

Mehra, A., Kilduff, M., \& Brass, D. (1998). At the margins: A distinctiveness approach to the social identity and social networks of underrepresented groups. Academy of Management Journal, 41, 441-452.

Moyerman, D., \& Forman, B. (1992). Acculturation and adjustment: A meta-analytic study. Hispanic Journal of Behavioral Sciences, 14, 163-200.

Phinney, J. (1990). Ethnic identity in adolescents and adults: Review of research. Psychological Bulletin, 108, 499-514.

Phinney, J. (1992). The multigroup ethnic identity measure: A new scale for use with adolescents and youth adults from diverse groups. Journal of Adolescent Research, 7, 156-176.

Phinney, J., \& Alipuria, L. (1996). At the interface of cultures: Multiethnic/multiracial high school and college students. Journal of Social Psychology, 136, 130-158.

Rhee, E., Uleman, J., Lee, H., \& Roman, R. (1995). Spontaneous self-descriptions and ethnic identities in individualistic and collectivistic cultures. Journal of Personality and Social Psychology, 69, 142-152.

Rogoff, B. (1990). Apprenticeship in thinking. New York: Oxford University Press.

Turner, J. C., Oakes, P. J., Haslam, S. A., \& McGarty, C. (1994). Self and collective: Cognition and social context. Psychological Bulletin, 20, 454-463. 